\title{
Green Fabrication of Thermal-stable oxidized Cellulose Nanocrystals by Evolutive Fenton Reaction and In-Situ Nanoreinforced Thermoplastic Starch
}

\section{Bingbing Gao}

South China University of Technology

Jiahui Yang

South China University of Technology

Shuidong Zhang ( $\nabla$ starch@scut.edu.cn )

South China University of Technology https://orcid.org/0000-0002-3973-2052

\section{Xiangyu Li}

South China University of Technology

\section{Research Article}

Keywords: Oxidized cellulose nanocrystals, thermal stability, "Carboxyl-Hydroxyl” hydrogen bonds, Fenton reaction

Posted Date: February 12th, 2021

DOI: https://doi.org/10.21203/rs.3.rs-221425/v1

License: (c) (1) This work is licensed under a Creative Commons Attribution 4.0 International License. Read Full License

Version of Record: A version of this preprint was published at Cellulose on July 13th, 2021. See the published version at https://doi.org/10.1007/s10570-021-04039-7. 


\section{Abstract}

High performances fiber and improved interfacial interaction can enhance the properties of polymer composites. Herein, microcrystalline cellulose (MCC) was oxidized by $\mathrm{H}_{2} \mathrm{O}_{2} / \mathrm{CuSO}_{4}$, a new Fenton process, to achieve oxidized MCC (OCNCs) with $16 \pm 1 \%$ carboxyl content. Noteworthy, the thermal stability of OCNC was superior to CNC prepared by acid hydrolysis. Interestingly, the primary alcohol groups of MCC were selective oxidized and OCNCs achieved $11.0 \mathrm{~nm}, 231.6 \mathrm{~nm}$ and $72 \%$ of average diameter, length and degree of crystallinity, respectively. Then glycerol, starch and OCNCs were reactive extruded to fabricate TPS/OCNC bionanocomposites and their structure and performances were evaluated systematically. Strikingly, significant improvement in glass transition temperature (from 63.1 to $94.5^{\circ} \mathrm{C}$ ) and notch impact strength (from 1.3 to $3.9 \mathrm{~kJ} / \mathrm{m}^{2}$ ) were noted for the amorphous TPS/OCNC with $1 \mathrm{wt} \%$ OCNC, and its tensile strength achieved $20.5 \mathrm{MPa}$, simultaneously. The improved mechanism of these performances was assigned to In-Situ forming "Carboxyl-Hydroxyl" hydrogen bonds which acted as the physically cross-linking interactions and improved the interfacial compatibility. We showcase Fenton reaction and reactive extrusion as the facile strategy to prepare sustainable and biodegradable TPS/OCNC bionanocomposites with properties more suitable for daily applications to replace petroleumbased plastic and eliminated the pollution of "microplastics."

\section{Introduction}

Presently, global warming and plastic pollution are the hot issues as the popular utilization of petroleumbased polymer products. A promising alternation approach is adopting the renewable and biodegradable materials, such as cellulose, starch, and so on, to substitute them. Recently, the development in nanostructured celluloses, or nanocellulose, has proposed due to the specific chemical and physical properties of these materials(Kargarzadeh et al. 2018). Cellulose nanocrystal (CNCs),contains a width of 3 to $50 \mathrm{~nm}$ and a length of 100 to $500 \mathrm{~nm}$ with very high crystallinity structure $\llbracket$ is a kind of rodlike and rigid nanoparticle extracted from natural biomass resources by acid treatment. This new environmentally friendly and renewable one-dimensional nanomaterial with light weight, displays excellent mechanical properties, large specific surface area, biodegradability and renewability(Eichhorn 2011; Habibi et al. 2010). Due to the attractive properties like high Young's modulus, crystallinity, specific surface area and aspect ratio, etc., CNCs have become an attractive component of nanomaterials and are widely used in the construction, pharmaceutical, and food stuff (Lu and Hsieh 2010; Mokhena and John 2020). When 1 wt \% of cellulose nanocrystals was incorporated, an 8-fold increase of polyurethane/cellulose nanocrystal nanocomposites in tensile strength was achieved. The reason was ascribed that CNCs could orient strongly at high strains and induce synergistic PU orientation effects. Whereas, a critical challenge to achieve the transfer of the exceptional mechanical properties of CNC-nanocomposites is the ability to achieve well-dispersed hydrophilic reinforcing CNC in hydrophobic polymer matrices, which further inhibited the interfacial compatibility in the composite systems(Chen et al. 2015). Another issue is the fabrication of CNC should be in a facile, green, fast, simple post-processing approach. For example, sulfuric acid hydrolysis is the most common method for the preparation of CNCs from original cellulose. 
Cellulose colloid suspension, which is the rudiment of current CNCs, was got from sulfuric acid degradation of cellulose and first reported by Ränby in 1949(Ranby 1949). Up to date, CNCs with ideal aspect ratio can be produced in a comparatively high yield maturely (Beck-Candanedo et al. 2005; Bondeson et al. 2006), the parameters were about $10 \mathrm{~nm}$ for diameter and $200 \sim 400 \mathrm{~nm}$ for length with a crystallinity of $63.3 \%$. However, the striking defect of this method is that the hydrolysis process is very fast and reduce the yields and degree of polymerization of CNC, meanwhile, the high concentration of sulfuric acid causes complex and hazardous post processing. Furthermore, sulfate groups cannot avoid being induced to the surface of CNCs products during hydrolysis with sulfuric acid, and it has been proved have negative effect on the thermostability of CNCs (Bao et al. 2021; Dufresne 2018; Roman and Winter 2004).

Several different methods have been developed for the preparation of CNCs in order to improve their thermal stability while reducing their impact on the environment. Huang's group focuses on using ball mills to fabricate CNCs (Huang et al. 2013; Kang et al. 2018), Li's group used the formic acid for preparing CNCs (Du et al. 2016; Li et al. 2015; Lv et al. 2019), and achieved fairly high yields and devising collection procedures to recover formic acid. However, although these methods could prepare CNCs with ideal size, they are limited by high prices or complex processing machine, which make them difficult to be used on an industrial scale(Yang et al. 2019).

In our previous studies, we developed a method for the selective preparation of high carboxyl content cellulose using Fenton reaction(Wen et al. 2019). This method is environmentally friendly and efficient, shows great potential for the preparation of CNCs(Koshani et al. 2018). However, the pretreatment of sodium hydroxide tends to lead to the drastic degradation of ORC in degree of polymerization (DP), diversified products and low yield with complex post-treatments, and therefore the method is required to avert. When microcrystalline cellulose (MCC) is oxidized by $\mathrm{H}_{2} \mathrm{O}_{2} / \mathrm{CuSO}_{4}$, what will occur for the oxidation process and the physical-chemical performance of $\mathrm{MCC}$ ? Incorporating physical crosslinking through hydrogen bonds to the TPS is a favorite approach to improve the comprehensive performances, and if the oxidized MCC (OMCC) with specific carboxyl content is incorporated into the hydrophilic polymer matrix with high tensity of hydrogen bonds in the low loading, as the ability of the carboxyl group for forming hydrogen-bonding ("Carboxyl-Hydroxyl group") between OCNC and starch is higher than hydroxyl groups ("hydroxyl-hydroxyl group")(Ghimire et al. 2020; Matsumoto et al. 2002; Ye et al. 2013), what are the variations of the new composites in structure and performances?

Thermoplastic starch (TPS) is a kind of biodegradable material made from polar plasticizers and starch through extrusion and other mechanical processing, which has the advantages of low price, biodegradable and edible(Alemdar and Sain 2008; Tavares et al. 2020). However, the weak mechanical properties and low glass transition temperature of the amorphous TPS is an urgent issue for improvement(Calvino et al. 2020). An efficient and convenient strategy to improve the properties of TPS is adopting the reinforcing fillers during the extrusion (Mittal et al. 2018; Zhang et al. 2007; Zhu et al. 2020). Among them, CNCs prepared by a simple method have many advantages to meet the growing demand for green and bio-based products (Chen et al. 2019; Nessi et al. 2019). Lu's group reported the 
introduction of cotton CNC to enhance plasticized starch(González et al. 2015) and found that the tensile strength and Young's modulus of the composites were increased by $212 \%$ and $736 \%$, respectively. Gonzalez et al. fabricated CNC by acidic hydrolysis and evaluated effects of the nanofiller content on the performances of TPS/CNC composites(González et al. 2020). Lourdes et al. successfully prepared nanocomposite films by mixing starch and cellulose nanocrystals(Coelho et al. 2020). To improve the CNC performances in polarity and hydrophobicity, surface pretreatment and chemical functionalization of CNCs had adopted(Huang et al. 2019). Consequently, all the presented research reveals that using environmentally friendly and effective methods to fabricate CNCs and their derivatives is a promising method to improve the performances of TPS, resulting in expanded application scope of TPS.

Herein, in this study, microcrystalline cellulose (MCC) was oxidized by Fenton process, which contained economical and environmentally friendly hydrogen peroxide and anhydrous copper sulfate, followed by a simple post-treatment to obtain oxidized CNCs (OCNCs) with diameters and lengths of $11 \mathrm{~nm}$ and $231.6 \mathrm{~nm}$, respectively. Interestingly, OCNCs with $17.1 \%$ carboxyl content achieved an improved thermal stability at $200{ }^{\circ} \mathrm{C}$ for $30 \mathrm{~min}$. Furthermore, when $1 \mathrm{wt} \%$ OCNCs was mixed with glycerol and corn starch to fabricate TPS/OCNCs composites via extrusion, a new relaxation peak to higher temperature was detected by dynamic mechanical analysis, noteworthy. These emerging phenomenon in thermal performances and the mechanism have not been reported and investigated. Undoubtedly, these variations in thermal performances of TPS/OCNCs composites would be related with the various hydrogen bonds (intermolecular interactions "Carboxyl-Hydroxyl group") when OCNCs was introduced to TPS. Simultaneously, after adding low OCNC loadings to TPS, the impact strength and tensile strength of the composites were $3.9 \mathrm{~kJ} / \mathrm{M}^{2}$ and $20.5 \mathrm{MPa}$, increased by $200 \%$ and $65 \%$ compared with TPS. The stronger reinforcing effect mechanism of OCNC for TPS was investigated by DMA, FT-IR, SEM, XRD and so on.

\section{Experimental Section}

\section{Materials}

Microcrystalline cellulose (MCC), starch, glycerol, $\mathrm{H}_{2} \mathrm{O}_{2}$ and $\mathrm{CuSO}_{4}$ were purchased from the Guangzhou Chemical Reagent Factory. All reagents were AR grade and used without further purification.

\section{Preparation of OCNCs}

Three sets of $20.0 \mathrm{~g} \mathrm{MCC}, 25.0 \mathrm{~mL}$ deionized water and $0.02 \mathrm{~g}$ copper(II) sulfate hydrate were added to three $250 \mathrm{~mL}$ erlenmeyer flasks, followed by the addition of $14.1 \mathrm{~g}, 42.2 \mathrm{~g}$ and $70.4 \mathrm{~g}$ of $30 \mathrm{w} \%$ hydrogen peroxide (molar ratios of hydrogen peroxide to cellulose monomers were 1: 1, 3:1 and 5:1, respectively).

The three flasks were placed in a thermostatic bath shaker at $35^{\circ} \mathrm{C}$. After 72 hours, the reaction solution was extracted, the precipitate was washed with deionized water, and the filtrate was centrifuged in a centrifuge at $8000 \mathrm{rpm}$ for 5 minutes. The three supernatants were mechanically processed in an 
ultrasonic cell crusher with 600 watts of power for 20 minutes to obtain a homogeneous $\mathrm{CNC} /$ cement slurry. After freeze-drying, the samples were labeled as OCNC-13.9, CNC3-17.1, and CNC-15.7, respectively.

\section{Preparation of TPS/OCNCs Composites}

Typical ingredients of TPS, 7 parts (weight) of starch and 3 parts (weight) of glycerol were added into a beaker. $500 \mathrm{~mL}$ OCNC-17.1/water slurry containing TPS with $0.5,1,2 \mathrm{wt} \%$ of OCNC-17.1 were prepared by the original OCNCs/water slurry and deionized water. Next, all the ingredients were mixed with starch and glycerol by mechanical stirring and heated into an even gel state. The gel was dried by oven under $105^{\circ} \mathrm{C}$ for $24 \mathrm{~h}$. Then the premixed sample was extruded by a double-screw extruder and cut into small pieces. Pure TPS and 1\% MCC/TPS samples were prepared in the same method by changing the OCNC-17.1 into other materials.

\section{Characterization}

The FT-IR spectra were recorded by Vector-33 FTIR spectrometer (Bruker, Germany) to characterize the structure of OCNCs and TPS/OCNCs composites. The resolution was $4 \mathrm{~cm}^{-1}$, and the scanning range was from 4000 to $500 \mathrm{~cm}^{-1}$. The X-ray diffraction (XRD) experiment was performed on an X'pert powder $X$-ray diffractometer over $2 \theta$ from 5 to $60^{\circ}$ with a scan rate of $5^{\circ} / \mathrm{min}$ at room temperature. The data of crystallinity (Xc) and FWHM were analyzed by Origin 8.0 software by deconvolution of diffraction patterns into peak areas for crystalline and amorphous components (French and Santiago Cintrón 2013; Ling et al. 2019; Nam et al. 2016).

\section{Morphological analysis}

Scanning electron microscopy (SEM) was performed by ZEISS Merlin field emission microscopy working at $10 \mathrm{kV}$ voltages to observe the morphology of the samples. Transmission electron microscopy (TEM) images were obtained by JEOL JEM-2100F at operating voltage of $200 \mathrm{kV}$. Samples were cryomicrotomed into about $100 \mathrm{~nm}$ thin sections at $-50^{\circ} \mathrm{C}$ by using a Lecia EM UC 6 system.

\section{Mechanical properties}

The granulated samples were dried at $80^{\circ} \mathrm{C}$ for $4 \mathrm{~h}$ and then injection molded into standard specimens. The V-impact notch specimens $\left(80 \times 10 \times 4 \mathrm{~mm}^{3}\right)$ of TPS were obtained by thermal press. Tensile properties, including tensile strength and elongation at the break were determined according to GB/T1040-2006 with a universal testing machine (104B, Shenzhen Wance Testing Machine Co., Ltd., China) at a crosshead speed of $20 \mathrm{~mm} / \mathrm{min}$. Impact strength was measured with an impact testing machines (501B-2, Shenzhen Wance Testing Machine Co.,Ltd., China) according to GB/T1843-2008. Five specimens of each sample were conducted and their mean value was reported mechanical properties.

\section{Dynamic thermomechanical analysis (DMA)}

DMA of all the samples were carried out on a NETZSCH DMA 242C dynamic mechanical analyzer (Germany) working in tensile mode. The test samples were fabricated by thermal press and tested from 100 to $100{ }^{\circ} \mathrm{C}$ at a heating rate of $5^{\circ} \mathrm{C} / \mathrm{min}^{-1}$ under a liquid nitrogen atmosphere and at a fixed frequency 
of $1 \mathrm{~Hz}$. The glass transition temperature $\left(\mathrm{T}_{\mathrm{g}}\right)$ was taken at the maximum of the tangent of the loss angle $(\tan \delta)$.

\section{Thermogravimetric Analysis (TGA)}

Composites samples were previously dried at $120^{\circ} \mathrm{C}$ for $4 \mathrm{~h}$ to remove the residual water in the samples and carried out immediately using a Perkin-Elmer Pyris thermal analyzer under nitrogen atmosphere at a heating rate of $10^{\circ} \mathrm{C} / \mathrm{min}$. The range of scanning temperature was from 50 to $600{ }^{\circ} \mathrm{C}$.

\section{Results And Discussion}

\section{The carboxyl content and DPv of OCNCs}

In our previous research(Wen et al. 2019), effects of hydrogen peroxide contents on the CC of ORC have been discussed in detail and concluded that both their CC and DPv are dominated by the molar ratio of hydrogen peroxide and RC. According to Roya et. al (Koshani et al. 2018), the reaction mechanism is that free radical ions generated by $\mathrm{H}_{2} \mathrm{O}_{2} / \mathrm{CuSO}_{4}$ penetrate to the outer layer of the crystalline regions and all amorphous regions. In this research, the CC and DPV of the OCNCs fabricated by different molar ratio of hydrogen peroxide and MCC are listed in Table 1. The data present that the CC of OCNC keep a stable value about $16 \pm 1 \%$ when the molar ratio of hydrogen peroxide and MCC increaseed from 1.0 to 3.0. When MCC does not suffer the pretreatment of $\mathrm{NaOH}$ solution, the oxidation just occurs in the surface of MCC, which is responded to the lower CC compared with those of ORC. The variations of the carboxyl group's introduction to the MCC surface endows them to repel each other and facilitates to generate the nanoscale of OCNC in aqueous solution, which is similar to the mechanism of action of the sulfate halfester groups on the CNC prepared by hydrolysis of sulfuric acid. Noteworthy, when the thermal stability and degradation are concerned, CNC contains carboxyl groups are more effective in polymer additives than sulfate halfester groups, which are too acidic and may break down the polymer matrix and cellulose itself (D'Acierno et al. 2020). The third column in the Table 1 shows that DPv of MCC was reduced from 235 to about 88 for OCNC with $15.7 \mathrm{CC}$. This result reveals that the oxidation and degradation of MCC occur simultaneously. However, the reduction of DPv is a normal phenomenon of CNC fabrication and it does not influence the properties of OCNC as the value is higher than 88 (the molecular weight of it is about $14300 \mathrm{~g} / \mathrm{mol}$ ), significantly. The destruction of mostly amorphous cellulose and partially crystalline cellulose occur during the preparation of CNCs which causes the DPv to decrease. Sulfuric acid hydrolysis, the most common method available on the market for the preparation of CNCs, also results in a final product with a DPv less than 100 (Alemdar and Sain 2008). 
Table 1

The carboxyl content and DPv of OCNCs

\begin{tabular}{|lll|}
\hline Samples & Carboxyl content (\%) & DPv \\
\hline MCC & 0 & 235 \\
\hline OCNC-13.9 & 13.9 & 128 \\
\hline OCNC-17.1 & 17.1 & 96 \\
\hline OCNC-15.7 & 15.7 & 88 \\
\hline
\end{tabular}

\section{Chemical structure analysis}

The main functional groups involved in the oxidation reaction is the hydroxyl groups in the glucose units, and the variation in groups after oxidation, such as the carboxyl group, can be characterized by FTIR spectra. The chemical structures of OCNC are characterized by FTIR, ${ }^{13} \mathrm{C}$-NMR and XRD (as shown in Fig. S1) and confirmed that OCNCs with different carboxyl content have been fabricated successfully by Fenton reaction when $\mathrm{CuSO}_{4}(0.1 \% \mathrm{wt}$ based on starch) acted as the catalyzer.

\section{Morphology of OCNCs}

Figure 1 gives the SEM micrographs of MCC and OCNCs. For MCC, it displays a fiber feature and its diameter ranges from 10 um to $30 \mathrm{um}$. Moreover, it also shows some cracks in the surface which may be assigned to the lower tensile strength and Young's modulus than those of OCNCs. It is reasonable to believe that this destroyed phenomenon on the fiber surface will be etched by the oxidant (Sang et al. 2017). Consequently, when $\mathrm{MCC}$ was oxidized by $\mathrm{H}_{2} \mathrm{O}_{2} / \mathrm{CuSO}_{4}$, it exhibits a different morphology as the degradation and oxidation for the MCC filer. The OCNCs show nanoscale size fibers with needle-like shape because the mother fibers of MCC are destroyed by the oxidation and release the uniformed CNC. SEM results confirmed that when MCC is oxidized by $\mathrm{H}_{2} \mathrm{O}_{2} / \mathrm{CuSO}_{4}$ in a mild condition with different $\mathrm{H}_{2} \mathrm{O}_{2}$ contraction, the OCNC with about $16 \%$ carboxyl group is achieved.

To evaluate the OCNC morphology, TEM micrographs of the OCNC-17.1 at magnifications ranges from $5 \mathrm{~K}$ to $25 \mathrm{~K}$ were provided in Fig. 2. They show the aggregation of OCNC-17.1 occurred due to the large specific surface area, small particle size, and multitudinous active hydroxyl groups on the surface.

Moreover, the needle-like shape fibers own the averaged length and diameter are of 231.6 and $11.0 \mathrm{~nm}$, respectively. Strikingly, OCNC-17.1 presents the fibrillar structure with the uniform in length, which is consistent with the SEM results.

Summarily, the results of FTIR, ${ }^{13} \mathrm{C}$ NMR, SEM, TEM and XRD confirmed the fact that the oxidation occurs on the surface of cellulose nanocrystals and OCNCs with special CC is fabricated via $\mathrm{H}_{2} \mathrm{O}_{2} / \mathrm{CuSO}_{4}$ solution in the mild condition.

\section{Thermostability of CNCs and OMCCs}


Due to its high Young's module, one of the most popular applications of OCNCs is as the strengthened fiber for improving the mechanical and thermal performances of polymers via thermal processes, such as the extrusion, internal mixer. Consequently, the high thermal stability of OCNCs is the key factor to fabricate improved properties of the polymer/OCNCs composites. Unfortunately, the traditional OCNCs produced by sulfuric acid hydrolysis have huge defects in thermostability, because the method will incorporate the sulfate groups on its surface, which will speed up the decomposition of both cellulose and polymer matrix under the high processing temperature (Rosa et al. 2010; Wang et al. 2020). According to our previous research, oxidized starch or cellulose with different carboxyl groups produced by hydrogen peroxide showed a higher carbon residue and lower toxic gas release than those of starch or cellulose (Peng et al. 2017; Zhang et al. 2015). Figure 3 shows the thermal behavior of MCC and OCNCs under different temperature and time. The $T_{5 \%}, T_{\max }$ and carbon residual under $600{ }^{\circ} \mathrm{C}$ of $\mathrm{MCC}$ and OCNCs achieved from Fig. 3a are listed in Table S2. They reveal that all samples show a one-step weight loss, the both $\mathrm{T}_{5 \%}$ and $\mathrm{T}_{\max }$ of OCNCs decrease with the increase of $\mathrm{CC}$, while the carbon residual increases. The incorporation of the carboxyl group in the glucose unit of cellulose will decompose firstly and cause OCNCs to occur decarboxylation. Meanwhile, the higher carboxyl groups also act a catalyzer to accelerate the thermal degradation of cellulose. Interestingly, the decomposing of carboxyl groups will construct an acid condition and promote glucose units to form higher carbon residual and change the original thermal decomposition of MCC. Consequently, OCNC-17.1 obtains an $18.7 \%$ carbon residual at $600{ }^{\circ} \mathrm{C}$, much higher than that of MCC.

To evaluate the thermal stability of OCNC-17.1 vary in the thermal process, isothermal TGA have been performed to simulate the temperature environment in internal mixer and the curves are presented at Fig. 3 (c, $d$ and e), and the values of residual mass of samples after 30 minutes are listed in Table S3. Due to the residual water in the sample, all of them lost about $5 \%$ weight at the first five minutes, then the samples diversify with the time increases. The curves reveal that MCC displays the highest thermal stability of all the samples, while OCNCs fabricated by sulfuric acid hydrolysis (CNC-S) exhibits the lowest value. When the temperature increases, the thermal stability of all samples reduce, which demonstrates that cellulose and its derivative will suffer a thermal decompose. Noteworthy, OCNC-17.1 achieves a higher thermal stability than that of CNC-S, which proves our hypothesis that when MCC is oxidized by $\mathrm{H}_{2} \mathrm{O}_{2} / \mathrm{CuSO}_{4}$, the physical-chemical performance of OCNCs in thermal stability improves, significantly. The improved thermal stability is very promising for adopting OCNC-17.1 to extrude or internal mix with thermoplastic starch without suffering the significant thermal stability. Consequently, much anticipated highlight of OCNC-17.1 mixed with TPS or other polymers, an improvement in thermal performance will achieve.

\section{DMA and Thermostability of composites}

The improved dynamic thermal properties of the nanocomposites are mainly due to the strong interaction between the TPS matrix and the additives through the formation of hydrogen bonding and the formation of permeation networks(Kargarzadeh et al. 2017). In this study, we used DMA to evaluate the compatibility of OCNC-17.1 with TPS composites and their thermal performances. The variations of 
storage modulus $\left(\mathrm{E}_{0}\right)$ and loss factor (tan $\delta$ ) of TPS, TPS/MCC and TPS/OCNC-17.1 with temperature and OCNC-17.1 content are shown in Fig. 4. Figure 4a shows that the $\mathrm{E}_{0}$ of all samples decreases significantly at around $-50^{\circ} \mathrm{C}$, which is the glass transition triggered by the addition of glycerol. The phenomenon is assigned the fact that glycerol acts as a plasticizer to increase the free volume or molecular mobility starch molecular chains by reducing the $\mathrm{H}$-bonds between the polymer chains (Canché-Escamilla et al. 2011; Cao et al. 2013). The storage modulus tends to decrease with increasing temperature as the amorphous TPS softens. Strikingly, the values of TPS/OCNC-17.1 is much higher than that of pure TPS and TPS/MCC, which indicated that the introduction of OCNC-17.1 can significantly improve the storage modulus of the blends when the temperature is lower than $50{ }^{\circ} \mathrm{C}$. Furthermore, the $\mathrm{E}_{0}$ of TPS/OCNC-17.1 composites significantly enhance with the increase of OCNC-17.1 contents and TPS/2 OCNC-17.1 achieves the highest value of all samples as the temperature is lower than $0{ }^{\circ} \mathrm{C}$. This improvement is attributed to the fact that the addition of OCNC-17.1 restricts the segmented motion of the starch segments, effectively improving the stiffness of TPS. Due to physical crosslinking through hydrogen bonds can hinder the starch macromolecular chains to motion. The virtue will motivate TPS/OCNC-17.1 to utilize in the room temperature environment.

Figure $4 \mathrm{~b}$ shows the temperature dependence of the loss factor (tan $\delta$ ) of TPS, TPS/MCC and TPS/OCNC-17.1 composites. For TPS, it shows a maximum value of tan $\delta$ at $63.1^{\circ} \mathrm{C}$ which attributes to glass transition temperature $\left(\mathrm{T}_{\mathrm{g}}\right)$ of the "starch rich phase," while the glass transition temperature of TPS $/ 1 \mathrm{MCC}$ increases to $76.9^{\circ} \mathrm{C}$, which indicates that the addition of MCC effectively improves the dynamic thermal performance of TPS. When MCC is introduced, it will form a new hydrogen bonds between cellulose and starch ("hydroxyl-hydroxyl" group) and obstruct the segmented motion of the starch segments, which responds to the increase of $T_{g}$. It is noteworthy that the TPS/OCNC-17.1 composites emerge a new peak at higher temperature $\left(\mathrm{T}_{\mathrm{g} 2}\right)$ when TPS is used as the control. When the content of OCNC-17. 1 ranges from $0.5-2 \%$, the $\mathrm{T}_{\mathrm{g} 2}$ of TPS/OCNC- 17.1 composites are $83.9{ }^{\circ} \mathrm{C}, 93.5^{\circ} \mathrm{C}$ and $89.2{ }^{\circ} \mathrm{C}$, respectively. The results demonstrate the additive OCNC-17.1 can construct a new structure with the TPS matrix through hydrogen bond interactions, such increased physical crosslinking through "Carboxyl-Hydroxyl group" hydrogen bonds, which made the TPS molecular chain more rigid. Furthermore, when the content of MCC and OCNC-17.1 is $1 \%$ for the composites, the hydrogen bond interactions between OCNC-17.1 and starch may be higher than that of MCC and starch due to the variations of hydrogen bond type. When carboxyl groups are introduced to cellulose, they can form a stronger cooperation between cellulose and starch and enhance the hydrogen bond ("Carboxyl-Hydroxyl group") interactions(Wang et al. 2019). Consequently, the cohesive energy density of TPS/OCNC-17.1 composites will be improved and resulting in improved thermal and mechanical performances.

The thermogravimetric curves of TPS, TPS/1 MCC and TPS/1 OCNC-17.1 under nitrogen atmosphere are presented in Fig. 4(c and d). By the TG curves, the initial and maximum temperature of the thermal degradation ( $\mathrm{T}_{5 \%}$ and $\mathrm{T}_{\text {max }}$ ) were determined, and their values are listed in Table S4. All samples display a one-step decomposed behavior with similar features of TPS, the $T_{5 \%}$ and $T_{\max }$ is about $200{ }^{\circ} \mathrm{C}$ and 300 ${ }^{\circ} \mathrm{C}$, respectively. When the temperature rises from 100 to $150{ }^{\circ} \mathrm{C}$, the weight loss of all samples is 
attributed to the evaporation of the residual water, then the starch suffers a slow thermal decomposition, such as dehydration, depolymerization and oxidation of hydroxyl groups, as the temperature increases from 150 to $250^{\circ} \mathrm{C}$. A drastic weight loss of all samples is observed when the temperature ranges from 250 to $350^{\circ} \mathrm{C}$. This phenomenon is assigned to both the glycerol evaporation (the shoulder peak near $280^{\circ} \mathrm{C}$ ) and the disruption of glucose units (the peak near $310^{\circ} \mathrm{C}$ ). Importantly, the scission of glycosidic bonds of starch will release some volatiles, such as $\mathrm{CO}_{2}, \mathrm{CO}, \mathrm{H}_{2} \mathrm{O}$, acetaldehyde, furan and 2-methyl furan(Yang et al. 2013), while only generate a small carbon residue. Interestingly, though the thermal stability of OCNC-17.1 is lower than that of MCC, TPS/ 1 OCNC-17.1 achieves the highest $T_{5 \%}$ value of all samples. According to the results of DMA and FT-IR, OCNC-17.1 can form a stronger cooperation due to the improved hydrogen bond interactions between the carboxyl group and hydroxyl group, which is responded to the improvement of $T_{5 \%}$ for TPS/1 OCNC-17.1. When the temperature elevates to higher value, the hydrogen bond interactions eliminate due to the dehydration and oxidation of hydroxyl groups. Consequently, all samples obtain the same $\mathrm{T}_{\max }$ value about $310^{\circ} \mathrm{C}$.

The TGA curves and $\mathrm{E}_{\mathrm{a}}$ data as a function of conversion of TPS and its composites are calculated by Flynn-Wall-Ozawa method (Fig. S3) and displayed in Fig. S2. Based on the results of DMA and TGA, it can be deduced that the improvement of $\mathrm{E}_{\mathrm{a}}$ is assigned to the stronger cooperation between the carboxyl group and hydroxyl group.

\section{FTIR and XRD analysis of the composites}

During the extrusion of TPS/OCNC, the hydrogen bonds among the starch, glycerol and OCNC molecules will be re-established. FT-IR measurement (presented in Fig S4a) reveals that formation of hydrogen bonding between the TPS and OCNC-17.1, which will enhance the intermolecular hydrogen bonds of the composites. Furthermore, by the results of XRD (presented in Fig S4b), the $X_{c}$ (degree of crystallinity) of TPS/1 OCNC-17.1 increases to $15.2 \%$ which may be assigned to the B-type crystallization(Putaux et al. 2000). It can be deduced that OCNC-17.1 may serve as the heterogeneous nucleation and promote the Btype crystallization due to its good compatibility with starch. According to the XRD results, it can be concluded that TPS/1 OCNC-17.1 is an amorphous polymer with $15 \%$ hybrid B-type and $\mathrm{V}_{\mathrm{H}^{-t y p e}}$ crystal(Brisson et al. 1991).

Generally, hydrogen bond interaction will be destroyed and leading hydroxyl group vibrations to a blue shift when the temperature increases. Herein, temperature-dependent FTIR measurements were used to study the temperature dependence of hydrogen bond interaction, Fig. 5 shows the temperature-dependent FTIR spectra of TPS and its composites. All the curves exhibit a broad-stronger absorption peak around $3300 \mathrm{~cm}^{-1}$, which is assigned to the $\mathrm{H}$-bonded hydroxyl groups in TPS (glycerol, $\mathrm{H}_{2} \mathrm{O}$ and starch). For TPS, the blue shift phenomenon cannot be discovered clearly when the temperature increases. $\mathrm{A}$ significant variation of the curve is that a peak around $1645 \mathrm{~cm}^{-1}$ emerges at $90{ }^{\circ} \mathrm{C}$ and its peak intensity increases with the temperature ranges from 90 to $150{ }^{\circ} \mathrm{C}$. For curves of TPS/ 1 OCNC-17.1 and TPS/2 OCNC-17.1, the absorption peak around $3458 \mathrm{~cm}^{-1}$ and $3441 \mathrm{~cm}^{-1}$ of the hydroxyl-stretching vibration is shifted blue by 4 and $13 \mathrm{~cm}^{-1}$, respectively, which indicates that the hydrogen bonding in the 
composites are disrupted with the increasing temperature. Noteworthy, the peak around $1645 \mathrm{~cm}^{-1}$ of TPS/1 OCNC-17.1 and TPS/2 OCNC-17.1emerges at 110 and $120^{\circ} \mathrm{C}$ around $1650 \mathrm{~cm}^{-1}$, and the peak intensity increases with the temperature ranges from 90 to $150^{\circ} \mathrm{C}$. The reason is due to the weakening of the interactions between hydrogen bonds with increasing temperature, resulting in the release of $\mathrm{OH}$ from the hydrogen bond network. Moreover, for TPS/2 OCNC-17.1, the peak feature for $3411 \mathrm{~cm}^{-1}$ changes from flat to increased intensity peak, which is ascribed to the reduction of associated hydrogen bonds. The results of Temperature-dependent FTIR spectra confirm that the formation of "Carboxyl-Hydroxyl" hydrogen bonds, and the introduction of OCNCs will motivate the ability of TPS to form improved hydrogen bonds force.

\section{Mechanical properties of thermoplastic starch composites with different cellulose products}

Cellulose has similar structure (same monomer) with starch, therefore, when the carboxyl group is introduced to the cellulose, it is expected that OCNCs will achieve good compatibility with TPS and show considerable improvement in mechanical performances. Based on the above data, OCNC-17.1 was chosen as the reinforcement fiber for TPS due to its high comprehensive performances. TPS was mixed with OCNC-17.1 with different contents in water, then they were extruded to fabricate TPS/OCNC-17.1 composites. Figure 6 shows the effect of OCNC-17.1 contents on the izod notched impact strength and tensile strength of TPS/OCNC-17.1 composites. The results reveal that incorporation of OCNC-17.1 with appropriate contents can simultaneously strengthen and toughen TPS matrix. Interestingly, the impact strength and tensile strength of TPS/1 OCNC-17.1 are $3.9 \mathrm{~kJ} / \mathrm{m}^{2}$ and $20.5 \mathrm{MPa}$, respectively, the increment values are $200 \%$ and $65 \%$ when TPS is used as the control.

In detail, impact strength of TPS/OCNC-17.1 composites range from 1.3 to $3.9 \mathrm{~kJ} / \mathrm{m}^{2}$ when OCNC-17.1 content rises from 0.5 to 1 . After that, the value reduces to $2.0 \mathrm{~kJ} / \mathrm{m}^{2}$ for TPS $/ 2$ OCNC-17.1. The impact strength of TPS/OCNC-17.1 composites are dominated by fiber dispersion and the adhesion between fiber and substrate interface(Sapkota et al. 2017). Since both TPS and OCNC-17.1 contain hydroxyl groups that can form hydrogen bonds between the interfaces, according to the results of DMA and FT-IR, they exist good interfacial adhesion between TPS and OCNC-17.1. Strong H-bonding between OCNC-17.1 and TPS interfaces promote to the formation of a rigid network of OCNC-17.1, which effectively strengthens the substrate. Generally, the mechanical properties of fiber reinforced thermoplastic composites are influenced by the length distribution of the fibers(Shuidong et al. 2014). According to the SEM, the average length (L) of OCNC17.1 is $231.6 \mathrm{~nm}$, while its average diameter (D) is $11.0 \mathrm{~nm}$, which employs these fibers to serve as reinforcement fillers for TPS. Similar with the results for impact strength, TPS/1 OCNC17.1 has the highest tensile strength, showing a $65 \%$ improvement relative to TPS. According to the results of XRD, the crystallinity of TPS/1 OCNC17.1 has not significant increased when compared with that of TPS. Consequently, the reason of improvements in mechanical performances can be assigned to the enhanced hydrogen bonds of OCNC-17.1 and the introduction of OCNC which reinforce TPS matrix. However, at higher OCNC-17.1 loadings, ranges from 1-2\%, both impact strength 
and tensile strength of the composites decrease, which may be assigned to the agglomeration of OCNC17.1 and deteriorate the dispersion of OCNC-17.1 in TPS matrix. The results can be evidenced by SEM which display in Fig S5.

As shown in Fig. 7, the improved dispersion may be attributed to OCNC-17.1 shows a nano-size fiber and own a larger aspect ratio than MCC. Importantly, due to the formation of enhanced hydrogen bonds between OCNC-17.1 and starch, the interface between OCNC and TPS is roast and tightly integrated, which achieve good phase compatibility. Consequently, the mechanical performances of TPS/1 OCNC17.1 achieve the highest values of all samples.

\section{Conclusions}

OCNC-17.1, a novel sustainable and biodegradable nano-fiber, is successfully prepared by Fenton reaction: microcrystalline cellulose (MCC) was oxidized by $\mathrm{H}_{2} \mathrm{O}_{2} / \mathrm{CuSO}_{4}$. Then this high thermal stability nano-fiber was reactive extruded with glycerol and starch to fabricate thermoplastic starch (TPS) based bionanocomposites with improved comprehensive performances. When 1\% OCNC-17.1 is incorporated to TPS, the significant improvements in glass transition temperature, notch impact strength and tensile strength increase from $63.1^{\circ} \mathrm{C}$ to $94.5^{\circ} \mathrm{C}, 1.3$ to $3.9 \mathrm{~kJ} / \mathrm{m}^{2}, 12.4$ to $20.5 \mathrm{MPa}$, respectively. When Temperature-dependent FTIR measurements, DMA, TGA, FTIR, XRD and SEM, are adopted to describe the improved mechanism of these performances, the analysis results reveal that the In-Situ formation of "Carboxyl-Hydroxyl" hydrogen bonds which serve as the physically cross-linking interactions and elevate the phase interfacial compatibility. When OCNC is introduced to TPS, it increases the hydrogen bonds variation and motivates TPS to form more sizes of intermolecular hydrogen bonds (just like the crosslinking density). Consequently, we propose oxidation and reactive extrusion here as the facile strategy to prepare cheap, sustainable and biodegradable TPS/OCNC bionanocomposites with high properties. Once they can replace petroleum-based plastic materials for daily applications, they are very promising for eliminating the pollution of "microplastics" and "White Pollution" to protect the earth environment.

\section{Declarations}

\section{Acknowledgments}

The authors wish to acknowledge the academic discussion from Prof. Baochun Guo and financial support of the National Natural Science Foundation of China (No. 51773068), SKL of Bio-Fibers and Eco-

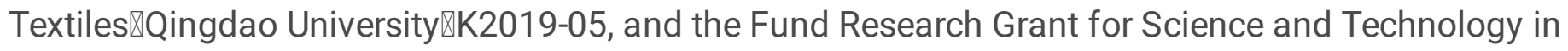
Guangzhou (202002030143), China, The Scientific and Technological Plan of Guangdong Province, China (No. 2019B090905005)".

\section{Notes}

The authors declare no competing financial interest. 


\section{References}

Alemdar A, Sain M (2008) Biocomposites from wheat straw nanofibers: Morphology, thermal and mechanical properties Composites Science and Technology 68:557-565

doi:https://doi.org/10.1016/j.compscitech.2007.05.044

Bao C et al. (2021) Extraction of cellulose nanocrystals from microcrystalline cellulose for the stabilization of cetyltrimethylammonium bromide-enhanced Pickering emulsions Colloids and Surfaces A: Physicochemical and Engineering Aspects 608:125442 doi:https://doi.org/10.1016/j.colsurfa.2020.125442

Beck-Candanedo S, Roman M, Gray DG (2005) Effect of reaction conditions on the properties and behavior of wood cellulose nanocrystal suspensions Biomacromolecules 6:1048-1054 doi:10.1021/bm049300p

Bondeson D, Mathew A, Oksman K (2006) Optimization of the isolation of nanocrystals from microcrystalline celluloseby acid hydrolysis Cellulose 13:171 doi:10.1007/s10570-006-9061-4

Brisson J, Chanzy H, Winter WT (1991) The crystal and molecular structure of VH amylose by electron diffraction analysis International Journal of Biological Macromolecules 13:31-39 doi:https://doi.org/10.1016/0141-8130(91)90007-H

Calvino C, Macke N, Kato R, Rowan SJ (2020) Development, processing and applications of bio-sourced cellulose nanocrystal composites Progress in Polymer Science 103:101221 doi:https://doi.org/10.1016/j.progpolymsci.2020.101221

Canché-Escamilla G, Canché-Canché M, Duarte-Aranda S, Cáceres-Farfán M, Borges-Argáez R (2011) Mechanical properties and biodegradation of thermoplastic starches obtained from grafted starches with acrylics Carbohydrate Polymers 86:1501-1508 doi:https://doi.org/10.1016/j.carbpol.2011.06.052

Cao X, Xu C, Liu Y, Chen Y (2013) Preparation and properties of carboxylated styrene-butadiene rubber/cellulose nanocrystals composites Carbohydrate Polymers 92:69-76 doi:https://doi.org/10.1016/j.carbpol.2012.09.054

Chen J, Chen F, Meng Y, Wang S, Long Z (2019) Oxidized microcrystalline cellulose improve thermoplastic starch-based composite films: Thermal, mechanical and water-solubility properties Polymer 168:228-235 doi:https://doi.org/10.1016/j.polymer.2019.02.026

Chen J, Lin N, Huang J, Dufresne A (2015) Highly alkynyl-functionalization of cellulose nanocrystals and advanced nanocomposites thereof via click chemistry Polymer Chemistry 6:4385-4395 doi:10.1039/C5PY00367A

Coelho CCdS, Silva RBS, Carvalho CWP, Rossi AL, Teixeira JA, Freitas-Silva O, Cabral LMC (2020) Cellulose nanocrystals from grape pomace and their use for the development of starch-based 
nanocomposite films International Journal of Biological Macromolecules 159:1048-1061 doi:https://doi.org/10.1016/j.ijbiomac.2020.05.046

D’Acierno F, Hamad WY, Michal CA, MacLachlan MJ (2020) Thermal Degradation of Cellulose Filaments and Nanocrystals Biomacromolecules 21:3374-3386 doi:10.1021/acs.biomac.0c00805

Du H, Liu C, Zhang Y, Yu G, Si C, Li B (2016) Preparation and characterization of functional cellulose nanofibrils via formic acid hydrolysis pretreatment and the followed high-pressure homogenization Industrial Crops and Products 94:736-745 doi:https://doi.org/10.1016/j.indcrop.2016.09.059

Dufresne A (2018) Cellulose nanomaterials as green nanoreinforcements for polymer nanocomposites Philosophical Transactions of the Royal Society A: Mathematical, Physical and Engineering Sciences 376:20170040 doi:10.1098/rsta.2017.0040

Eichhorn SJ (2011) Cellulose nanowhiskers: promising materials for advanced applications Soft Matter 7:303-315 doi:10.1039/COSM00142B

French AD, Santiago Cintrón M (2013) Cellulose polymorphy, crystallite size, and the Segal Crystallinity Index Cellulose 20:583-588 doi:10.1007/s10570-012-9833-y

Ghimire G et al. (2020) Influences of Hydrogen Bonding-Based Stabilization of Bolaamphiphile Layers on Molecular Diffusion within Organic Nanotubes Having Inner Carboxyl Groups Langmuir 36:6145-6153 doi:10.1021/acs.langmuir.0c00556

González K, Iturriaga L, González A, Eceiza A, Gabilondo N (2020) Improving mechanical and barrier properties of thermoplastic starch and polysaccharide nanocrystals nanocomposites European Polymer Journal 123:109415 doi:https://doi.org/10.1016/j.eurpolymj.2019.109415

González K, Retegi A, González A, Eceiza A, Gabilondo N (2015) Starch and cellulose nanocrystals together into thermoplastic starch bionanocomposites Carbohydrate Polymers 117:83-90 doi:https://doi.org/10.1016/j.carbpol.2014.09.055

Habibi Y, Lucia LA, Rojas OJ (2010) Cellulose Nanocrystals: Chemistry, Self-Assembly, and Applications Chemical Reviews 110:3479-3500 doi:10.1021/cr900339w

Huang L et al. (2019) Properties of thermoplastic starch films reinforced with modified cellulose nanocrystals obtained from cassava residues New Journal of Chemistry 43:14883-14891 doi:10.1039/C9NJ02623A

Huang P, Wu M, Kuga S, Huang Y (2013) Aqueous pretreatment for reactive ball milling of cellulose Cellulose 20:2175-2178 doi:10.1007/s10570-013-9940-4

Kang X, Kuga S, Wang C, Zhao Y, Wu M, Huang Y (2018) Green Preparation of Cellulose Nanocrystal and Its Application ACS Sustainable Chemistry \& Engineering 6:2954-2960

Page 14/21 
doi:10.1021/acssuschemeng.7b02363

Kargarzadeh $\mathrm{H}$ et al. (2018) Recent developments in nanocellulose-based biodegradable polymers, thermoplastic polymers, and porous nanocomposites Progress in Polymer Science 87:197-227 doi:https://doi.org/10.1016/j.progpolymsci.2018.07.008

Kargarzadeh H, Johar N, Ahmad I (2017) Starch biocomposite film reinforced by multiscale rice husk fiber Composites Science and Technology 151:147-155 doi:https://doi.org/10.1016/j.compscitech.2017.08.018

Koshani R, van de Ven TGM, Madadlou A (2018) Characterization of Carboxylated Cellulose Nanocrytals Isolated through Catalyst-Assisted $\mathrm{H}_{2} \mathrm{O}_{2}$ Oxidation in a One-Step Procedure Journal of Agricultural and Food Chemistry 66:7692-7700 doi:10.1021/acs.jafc.8b00080

Li B et al. (2015) Cellulose nanocrystals prepared via formic acid hydrolysis followed by TEMPOmediated oxidation Carbohydrate Polymers 133:605-612 doi:https://doi.org/10.1016/j.carbpol.2015.07.033

Ling Z et al. (2019) Effects of ball milling on the structure of cotton cellulose Cellulose 26:305-328 doi:10.1007/s10570-018-02230-x

Lu P, Hsieh Y-L (2010) Preparation and properties of cellulose nanocrystals: Rods, spheres, and network Carbohydrate Polymers 82:329-336 doi:https://doi.org/10.1016/j.carbpol.2010.04.073

Lv D et al. (2019) Tailored and Integrated Production of Functional Cellulose Nanocrystals and Cellulose Nanofibrils via Sustainable Formic Acid Hydrolysis: Kinetic Study and Characterization ACS Sustainable Chemistry \& Engineering 7:9449-9463 doi:10.1021/acssuschemeng.9b00714

Matsumoto A, Ueda A, Aota H, Ikeda J-i (2002) Effect of hydrogen bonds on intermolecular crosslinking reaction by introduction of carboxyl groups in free-radical crosslinking monomethacrylate/dimethacrylate copolymerizations European Polymer Journal 38:1777-1782 doi:https://doi.org/10.1016/S00143057(02)00055-1

Mittal G, Rhee KY, Mišković-Stanković V, Hui D (2018) Reinforcements in multi-scale polymer composites: Processing, properties, and applications Composites Part B: Engineering 138:122-139 doi:https://doi.org/10.1016/j.compositesb.2017.11.028

Mokhena TC, John MJ (2020) Cellulose nanomaterials: new generation materials for solving global issues Cellulose 27:1149-1194 doi:10.1007/s10570-019-02889-w

Nam S, French AD, Condon BD, Concha M (2016) Segal crystallinity index revisited by the simulation of Xray diffraction patterns of cotton cellulose $I \beta$ and cellulose II Carbohydrate Polymers 135:1-9 doi:https://doi.org/10.1016/j.carbpol.2015.08.035 
Nessi V et al. (2019) Cellulose nanocrystals-starch nanocomposites produced by extrusion: Structure and behavior in physiological conditions Carbohydrate Polymers 225:115123 doi:https://doi.org/10.1016/j.carbpol.2019.115123

Peng H, Zhang S, Yin Y, Jiang S, Mo W (2017) Fabrication of c-6 position carboxyl regenerated cotton cellulose by $\mathrm{H}_{2} \mathrm{O}_{2}$ and its promotion in flame retardency of epoxy resin Polymer Degradation and Stability 142:150-159 doi:https://doi.org/10.1016/j.polymdegradstab.2017.05.026

Putaux J-L, Buléon A, Chanzy H (2000) Network Formation in Dilute Amylose and Amylopectin Studied by TEM Macromolecules 33:6416-6422 doi:10.1021/ma000242j

Ranby B (1949) Aqueous colloidal solutions of cellulose micelles vol 3. Munksgaard Int Publ Ltd 35 Norre Sogade, Po Box 2148, DK-1016 Copenhagen, Denmark, doi:https://doi.org/10.3891/acta.chem.scand.030649

Roman M, Winter WT (2004) Effect of Sulfate Groups from Sulfuric Acid Hydrolysis on the Thermal Degradation Behavior of Bacterial Cellulose Biomacromolecules 5:1671-1677 doi:10.1021/bm034519+

Rosa MF et al. (2010) Cellulose nanowhiskers from coconut husk fibers: Effect of preparation conditions on their thermal and morphological behavior Carbohydrate Polymers 81:83-92 doi:https://doi.org/10.1016/j.carbpol.2010.01.059

Sang X, Qin C, Tong Z, Kong S, Jia Z, Wan G, Liu X (2017) Mechanism and kinetics studies of carboxyl group formation on the surface of cellulose fiber in a TEMPO-mediated system Cellulose 24:2415-2425 doi:10.1007/s10570-017-1279-9

Sapkota J, Natterodt JC, Shirole A, Foster EJ, Weder C (2017) Fabrication and Properties of Polyethylene/Cellulose Nanocrystal Composites Macromolecular Materials and Engineering 302:1600300 doi:https://doi.org/10.1002/mame.201600300

Shuidong Z, Lingcao T, Jizhao L, Hanxiong H, Guo J (2014) Relationship between structure and properties of reprocessed glass fiber reinforced flame retardant poly(butylene terephthalate) Polymer Degradation and Stability 105:140-149 doi:https://doi.org/10.1016/j.polymdegradstab.2014.04.009

Tavares KM, Campos Ad, Luchesi BR, Resende AA, Oliveira JEd, Marconcini JM (2020) Effect of carboxymethyl cellulose concentration on mechanical and water vapor barrier properties of corn starch films Carbohydrate Polymers 246:116521 doi:https://doi.org/10.1016/j.carbpol.2020.116521

Wang D, Yang T, Li J, Zhang J, Yu J, Zhang X, Zhang J (2020) Thermostable and Redispersible Cellulose Nanocrystals with Thixotropic Gelation Behavior by a Facile Desulfation Process ACS Sustainable Chemistry \& Engineering 8:11737-11746 doi:10.1021/acssuschemeng.0c03838 
Wang $Y$ et al. (2019) Hydrogen bonding derived self-healing polymer composites reinforced with amidation carbon fibers Nanotechnology 31:025704 doi:10.1088/1361-6528/ab4743

Wen J, Yin Y, Peng X, Zhang S (2019) Using $\mathrm{H}_{2} \mathrm{O}_{2}$ to selectively oxidize recyclable cellulose yarn with high carboxyl content Cellulose 26:2699-2713 doi:10.1007/s10570-018-2217-1

Yang Y, Chen Z, Zhang J, Wang G, Zhang R, Suo D (2019) Preparation and Applications of the Cellulose Nanocrystal International Journal of Polymer Science 2019:1767028 doi:10.1155/2019/1767028

Yang Z, Liu X, Yang Z, Zhuang G, Bai Z, Zhang H, Guo Y (2013) Preparation and formation mechanism of levoglucosan from starch using a tubular furnace pyrolysis reactor Journal of Analytical and Applied Pyrolysis 102:83-88 doi:https://doi.org/10.1016/j.jaap.2013.03.012

Ye H-I, Liu Y-f, Zhang X-h, Di D-I (2013) DFT study on hydrogen-bonding adsorption mechanism of rutin onto macroporous adsorption resins functionalized with amino, hydroxyl, and carboxyl groups Structural Chemistry 24:1443-1449 doi:10.1007/s11224-012-0174-0

Zhang Q-X, Yu Z-Z, Xie X-L, Naito K, Kagawa Y (2007) Preparation and crystalline morphology of biodegradable starch/clay nanocomposites Polymer 48:7193-7200 doi:https://doi.org/10.1016/j.polymer.2007.09.051

Zhang S, Liu F, Peng H, Peng X, Jiang S, Wang J (2015) Preparation of Novel c-6 Position Carboxyl Corn Starch by a Green Method and Its Application in Flame Retardance of Epoxy Resin Industrial \& Engineering Chemistry Research 54:11944-11952 doi:10.1021/acs.iecr.5b03266

Zhu J et al. (2020) Lightweight, High-Strength, and Anisotropic Structure Composite Aerogel Based on Hydroxyapatite Nanocrystal and Chitosan with Thermal Insulation and Flame Retardant Properties ACS Sustainable Chemistry \& Engineering 8:71-83 doi:10.1021/acssuschemeng.9b03953

\section{Figures}
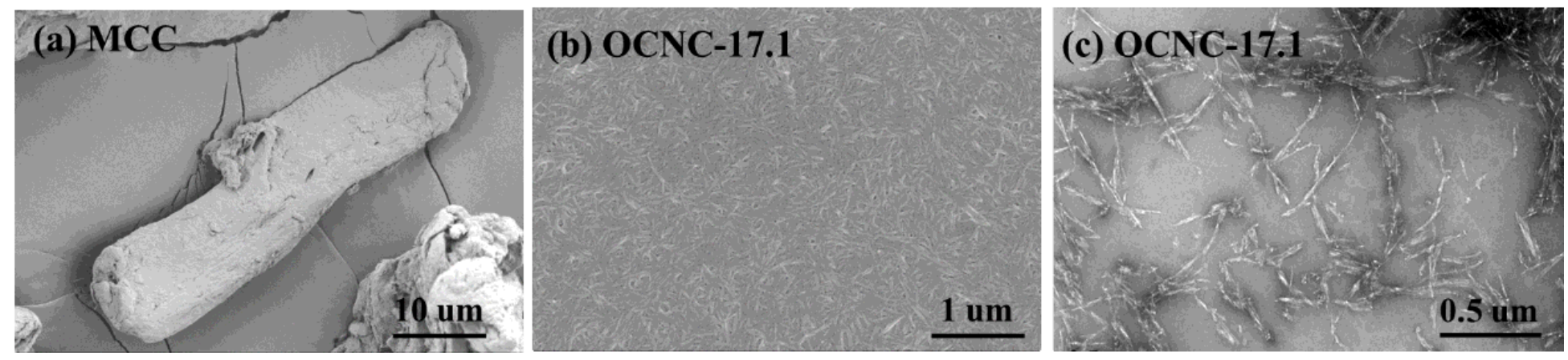

\section{Figure 1}

SEM micrographs of MCC and OCNC-17.1 

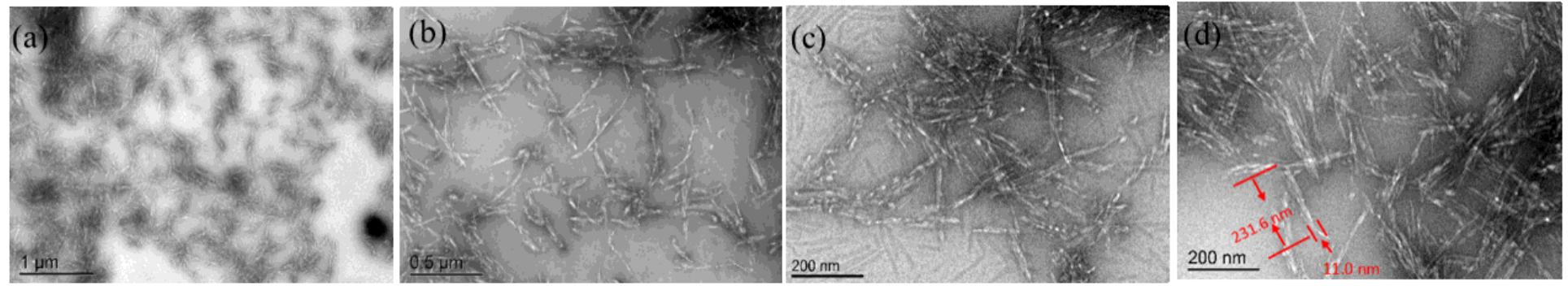

Figure 2

TEM of CNC-17.1 at magnifications ranges from $5 \mathrm{~K}$ to $25 \mathrm{~K}$

(a)

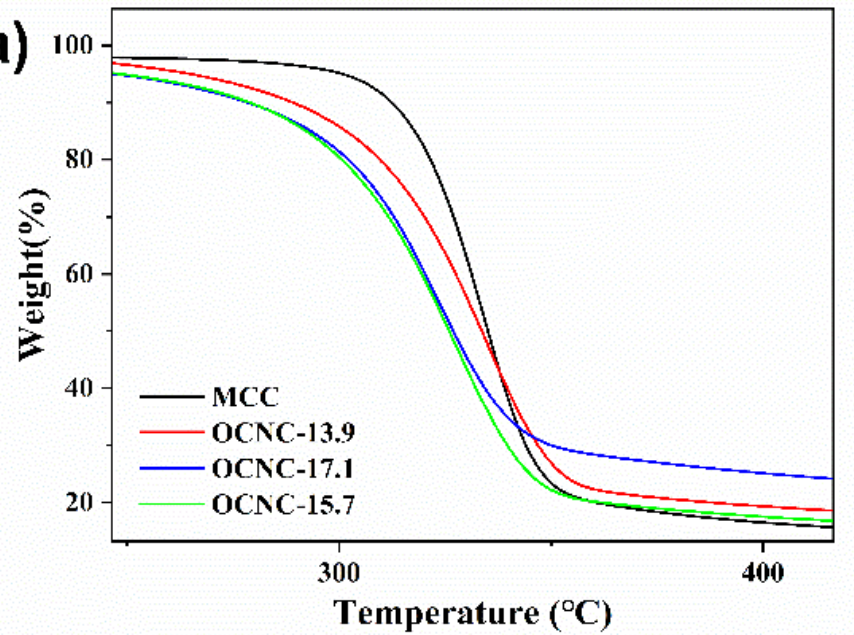

(c)

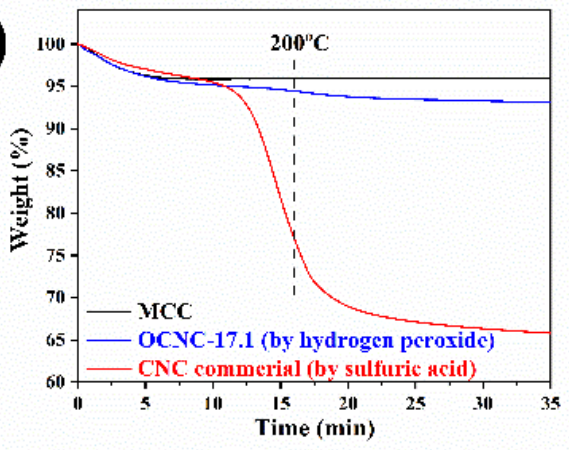

(d)

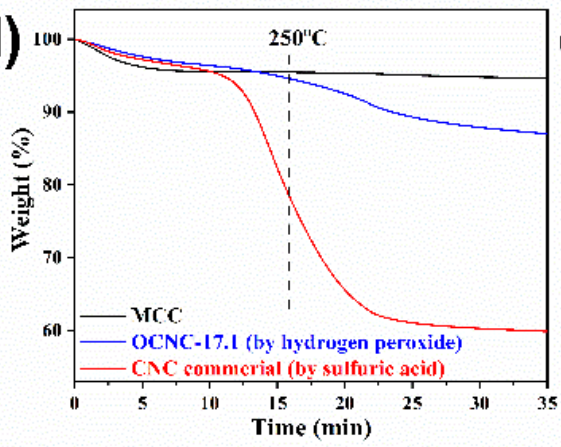

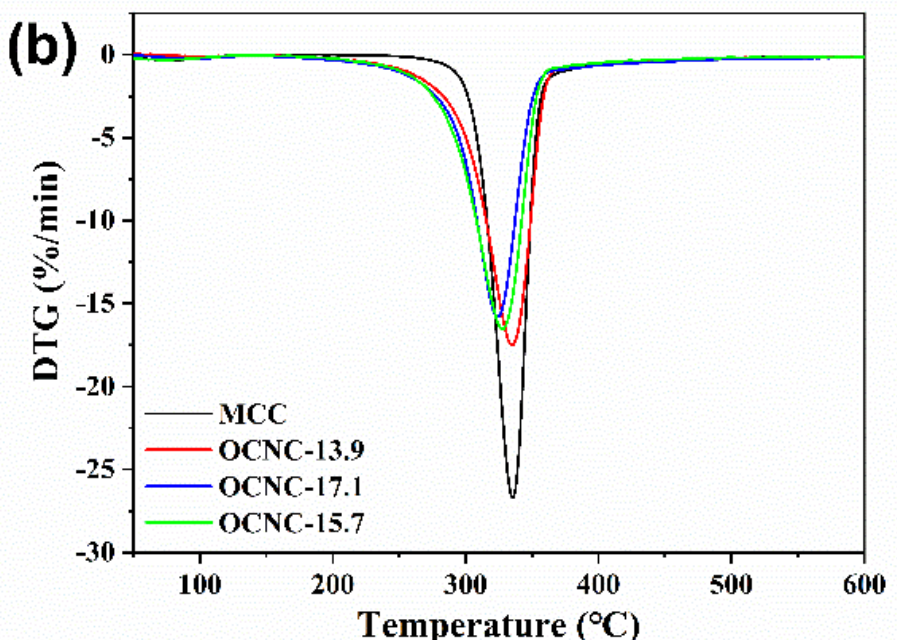

Temperature $\left({ }^{\circ} \mathrm{C}\right)$

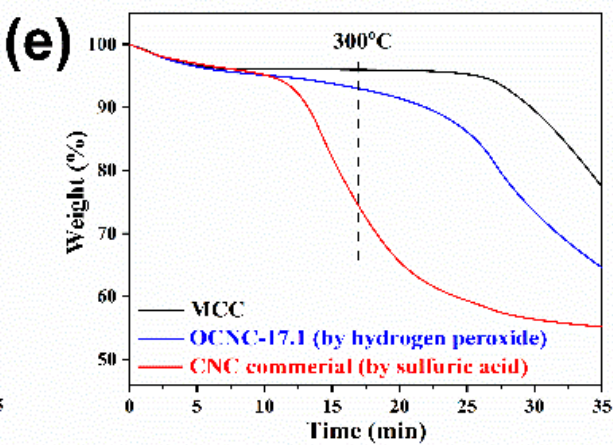

Figure 3

(a) TG curves of MCC and OCNCs (b) DTG curves of MCC and OCNCs, and isothermal TG of CNC commercial and OCNC-17. 1 in $200^{\circ} \mathrm{C}$ (c), $250{ }^{\circ} \mathrm{C}(\mathrm{d})$, and $300^{\circ} \mathrm{C}(\mathrm{e})$ 

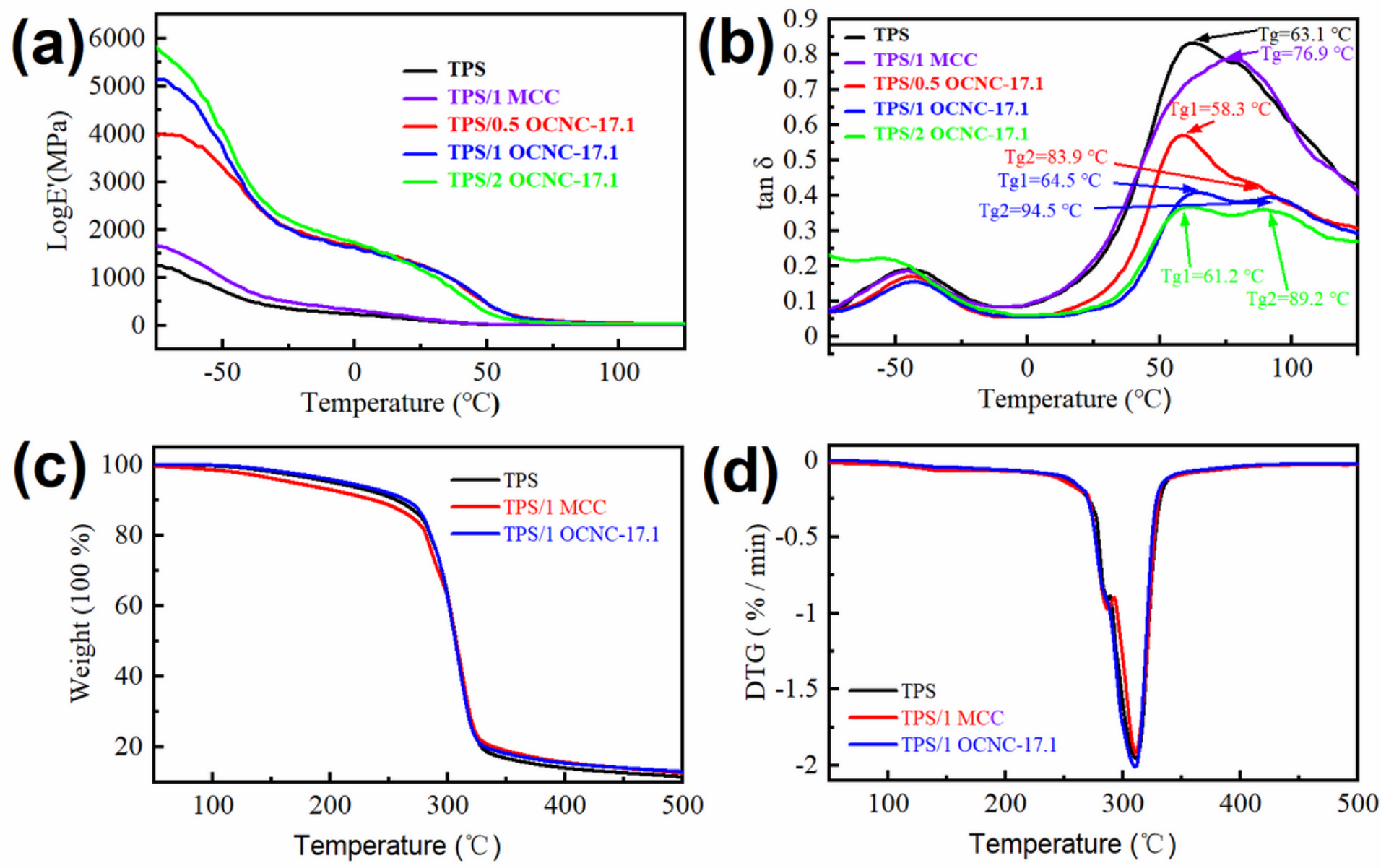

Figure 4

DMA and TGA for TPS/OCNC composites (a) The storage modulus (E0) (b) The loss factor (tan $\delta$ ) (c) TG curves of the composites (d) DTG curves of the composites 

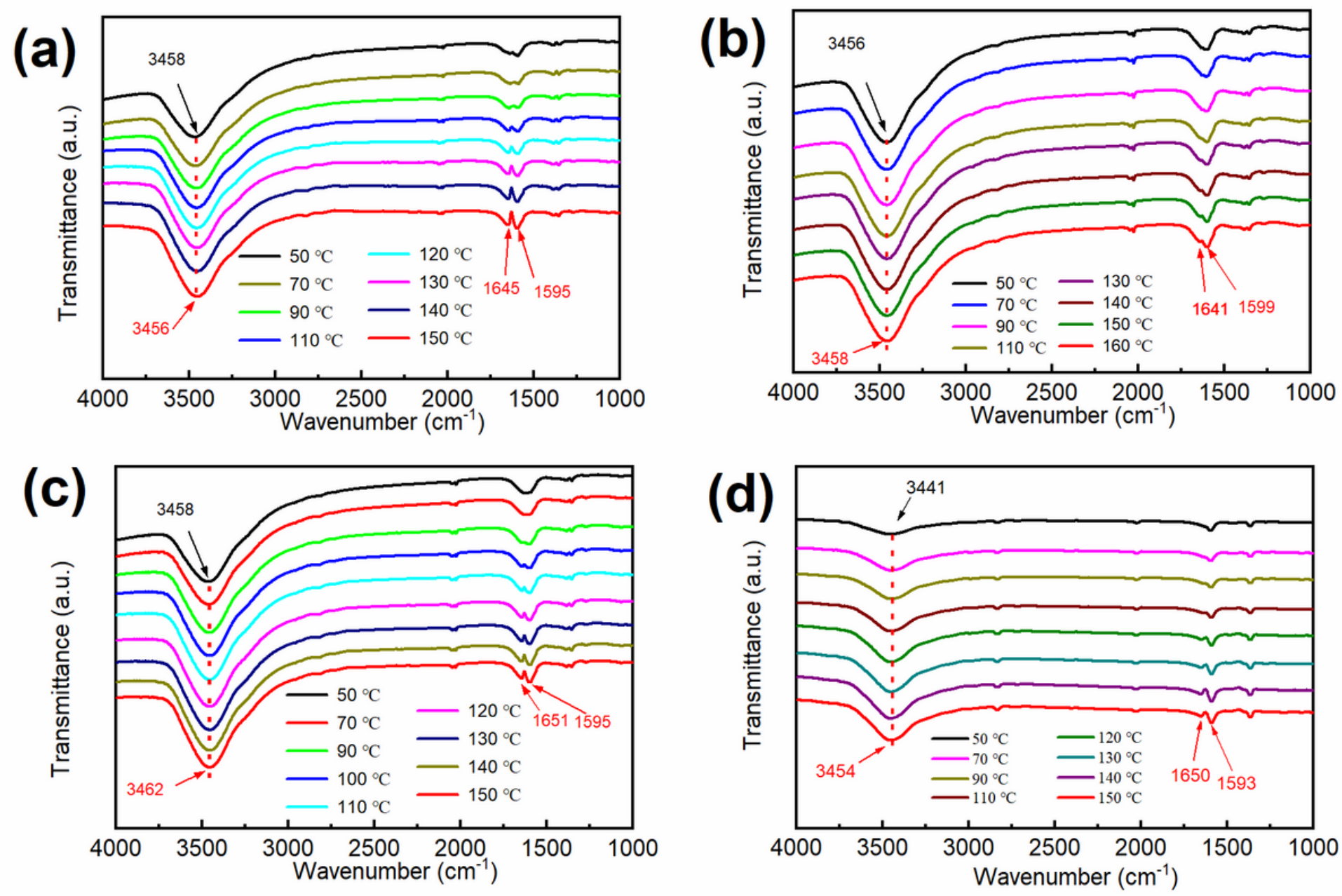

Figure 5

Temperature-dependent FTIR spectra of (a) TPS and (b) TPS/1 MCC (c) TPS/1 OCNC-17.1 (d) TPS/2 OCNC-17.1
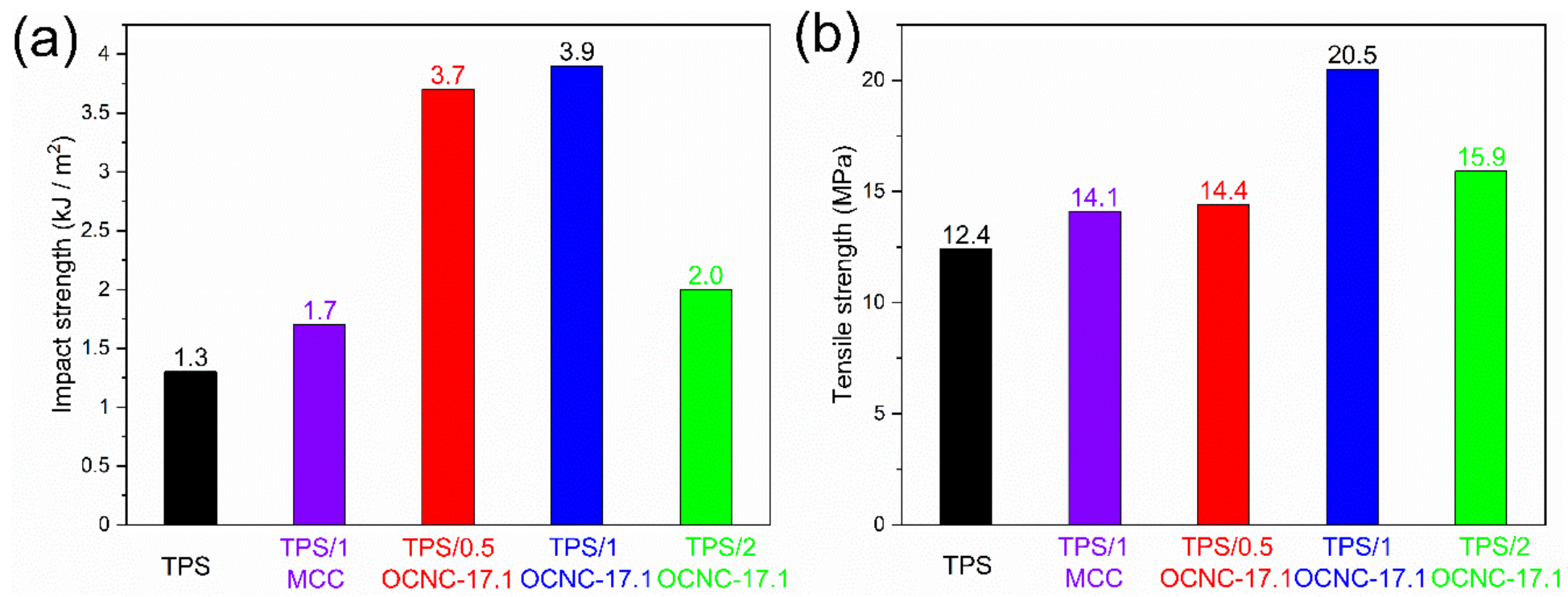
Figure 6

The effect of OCNC-17.1 contents on the (a) izod notched impact strength and (b) tensile strength of TPS/OCNC-17.1 composites
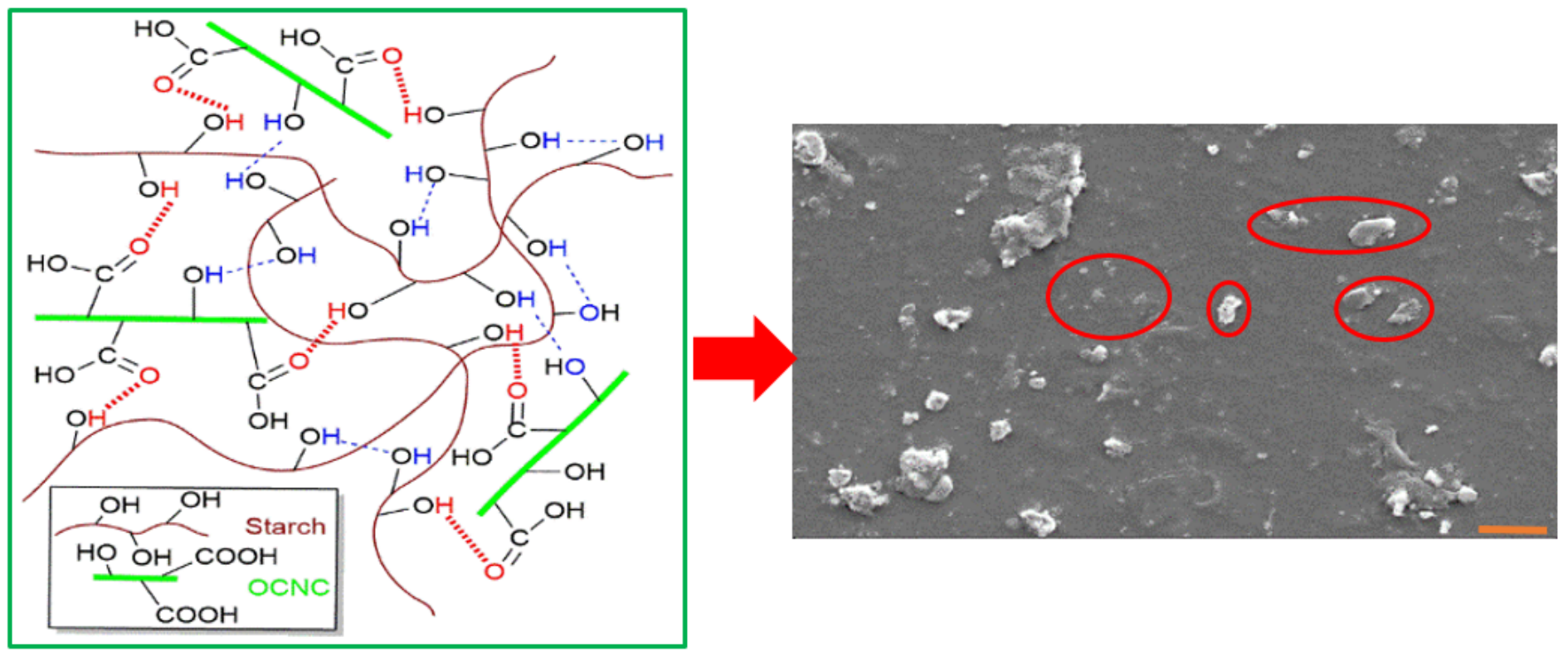

Figure 7

The reinforcement mechanism of TPS/OCNC composites

\section{Supplementary Files}

This is a list of supplementary files associated with this preprint. Click to download.

- SI20210111submissionplaintxt.docx 